\title{
Le lean : pensée et impensé d'une activité sans relâchement
}

Lean: Thoughts and oversights in respect of relentless activity

\section{Pascal Ughetto}

\section{(2) OpenEdition}

\section{Journals}

Édition électronique

URL : http://journals.openedition.org/activites/453

DOI : 10.4000/activites.453

ISSN : 1765-2723

\section{Éditeur}

ARPACT - Association Recherches et Pratiques sur les ACTivités

\section{Référence électronique}

Pascal Ughetto, "Le lean : pensée et impensé d'une activité sans relâchement », Activités [En ligne], 9-2 | Octobre 2012, mis en ligne le 15 octobre 2012, consulté le 19 avril 2019. URL : http:// journals.openedition.org/activites/453; DOI : 10.4000/activites.453

\section{(9) $\odot \Theta \Theta$}

Activités est mis à disposition selon les termes de la licence Creative Commons Attribution - Pas d'Utilisation Commerciale - Pas de Modification 4.0 International. 


\title{
Le lean : pensée et impensé d'une activité sans relâchement
}

\author{
Pascal Ughetto \\ Université Paris-Est, LATTS - 5, boulevard Descartes, Champs-sur-Marne \\ 77454 Marne-la-Vallée cedex 2 - pascal.ughetto@univ-paris-est.fr
}

\begin{abstract}
Lean: Thoughts and oversights in respect of relentless activity. Lean production has made a comeback in businesses since the mid2000s, triggering much debate about its impact on working practices. This paper seeks to highlight the manner in which "lean" conceives work. However, neither Ohno, who devised the method and its tools, nor Womack, Jones or Roos, its theoreticians, actually provide us with very much explicit theory. The key idea is to combat waste in the day-to-day operation of workshops. This may lead either to a reassessment of employees' working conditions or to an obsession with reducing "unproductive" work time by forcing human beings to constantly adapt. This relative uncertainty is all the more important insofar as its implementation outside the automotive sector means that it may be applied in very different productive contexts.
\end{abstract}

\section{KEYWORDS}

Lean, Lean management, Lean manufacturing, work

\section{Introduction}

Le lean a fait son retour et, avec lui, l'interrogation sur ses implications pour le travail. Le besoin d'analyses se fait particulièrement sentir du côté de l'ergonomie (Bourgeois \& Gonon, 2010), confrontée à des demandes d'accompagnement formulées par les directions d'entreprises ou les comités d'hygiène, de sécurité et des conditions de travail alors même que les conséquences pour l'activité font l'objet d'une forte incertitude : que fait le lean au travail; y a-t-il des espaces d'adaptation pour tenir compte des exigences du travail; ou renforce-t-il au contraire, purement et simplement, les contraintes de tous ordres, posturales, de gestion des conflits de buts ou autres ; favorise-t-il les troubles musculo-squelettiques ? Pour l'intervention ergonomique, cela est décisif puisqu'il s'agit de décider si elle permettra ou non de contribuer à des ajustements mutuels entre les dispositifs d'organisation et techniques et les sujets au travail et si l'ergonome sera un acteur de cet ajustement ou un agent du renforcement des contraintes. Derrière cela, l'interrogation qui taraude l'ergonome est de savoir si le lean est une mécanique implacable ou s'il laisse des choix ouverts pour la prise en compte des exigences du travail. La profession peut être partagée entre le fait d'y voir une méthode où il est tout à fait loisible d'introduire des modifications, même très simples, pour soulager les opérateurs, par exemple de contraintes posturales par le biais de bras artificiels pour des opérateurs intervenant sous les caisses sur une ligne de montage automobile, ou au contraire de la voir comme ayant fondamentalement tendance à durcir l'intensité du travail. Cela n'est pas sans réactiver les tensions internes sur la façon dont l'ergonomie - et quel type d'ergonomie - pouvait ou devait participer au déploiement des organisations tayloriennes et fordiennes. 
La recherche en sciences sociales ne s'est pas encore montrée des plus actives pour fournir des résultats sur la phase actuelle de développement du lean, après l'intérêt porté au «toyotisme» ou à la «lean production» durant les années 1980 et 1990. Si les méthodes observées chez Toyota avaient, à l'époque, acquis une renommée internationale et valeur de modèle à suivre, et s'il avait été décidé par les chercheurs de considérer comme particulièrement décisif de statuer sur la rupture qu'elles représentaient ou non vis-à-vis du taylorisme-fordisme, ces mêmes chercheurs ont laissé le débat s'épuiser depuis, plus par l'effet d'un désintérêt et le passage à d'autres objets que par la résolution de l'énigme.

Le lean faisant de nouveau parler de lui depuis le milieu des années 2000, l'énigme renaît : est-ce une machine compactant irrépressiblement le temps de travail ? Ou peut-on y voir une méthode préservant ou favorisant des espaces d'attention au travail et à la régulation des tensions de l'activité ? Des recherches commencent à apparaître sur les effets du lean sur la santé (Bertrand et Stimec, 2011 ; Bouville, 2010 ; Morvan, François et Bourgeois, 2008 ; Ughetto, 2011). Mais la trajectoire suivie par le lean et sa résurgence actuelle incitent, par ailleurs, à se poser la question de l'identité du lean, de la stabilité ou non de ce qu'il recouvre.

Dans cet article, il s'agira de se demander quelle représentation du travail, voire plus spécifiquement de l'activité de travail, est celle du lean. Sur quelle idée de l'activité le lean se fonde-t-il, quelle part y accorde-t-il, quelle théorie s'en fait-il ? On verra que la présence de la réflexion sur le travail n'est peut-être pas aussi immédiate et centrale que ce à quoi on a pu s'habituer avec la doctrine de Taylor. Mais, dans l'enquête à mener, il va falloir en passer par la question de ce qu'est le lean. Une partie de la thèse de cet article sera alors d'inviter à ne pas le substantialiser et de prendre le temps de regarder de près ce qu'il est, éventuellement de façon plurielle. Ce n'est que de loin, dans un regard rapide, que son identité paraît simple. De près, les choses s'avèrent plus compliquées, et donc aussi plus ouvertes, en particulier pour le travail.

\section{1.- Le lean, quel lean ?}

Depuis quelques années, les milieux du conseil aux entreprises et les acteurs managériaux ont manifesté un intérêt grandissant pour un ensemble de méthodes et d'outils communément appelé le lean. On assiste à leur diffusion dans les entreprises, non seulement de l'industrie, mais également des services, voire dans l'administration publique. Ces méthodes et ces outils paraissent appelés à avoir de réelles conséquences pour le travail. Évaluer la relation entre méthodes et outils lean, d'un côté, et le travail, de l'autre, incite à vouloir identifier «le lean» dans ses caractéristiques fondamentales, quasiment dans son être profond, et à lui rechercher une identité stable. Les confrontations à des cas concrets d'application du lean (Beauvallet et Huy, 2009 ; Bertrand et Stimec, 2011 ; Bouville, 2010 ; Colin, Grasser et Jacquot, 2011 ; Ughetto, 2009) conduisent cependant immédiatement à s'interroger sur une telle substance du lean.

\section{1.- Se garder d'une définition substantialiste}

Le lean a fait un retour récent, que l'on peut situer approximativement au milieu des années 2000 et connaît, dans cette phase, une diffusion qui l'amène à déborder des chaînes de montage de l'automobile qui étaient son domaine d'application premier. C'est probablement là l'une des principales ruptures avec la phase de diffusion des années 1980 et 1990, où il paraissait à peu près évident que la lean production concernait essentiellement les constructeurs automobiles et l'industrie de série. Aujourd'hui, le lean se fait valoir auprès des dirigeants d'entreprises en prétendant leur convenir quel que soit le domaine d'activité des firmes qu'ils dirigent. On trouve mention du lean et de l'intérêt de son développement dans des domaines parfaitement improbables au regard de la façon dont on comprenait la lean production les années 1990 : les administrations publiques, les hôpitaux, etc. Le lean se 
veut dorénavant une méthode générale, tout terrain, garantissant la révélation de gaspillages ordinaires porteurs de coûts sans addition de valeur pour le client; il permettrait donc des manières de fonctionner produisant autant ou plus avec moins.

Dès lors, si le contexte qui justifie de se pencher sur le lean est l'extension qu'il prend désormais, il faut prêter attention à ce qu'implique le fait qu'il s'échappe de son contexte de sa configuration productive (du Tertre, 1995) - d'origine. Du moins est-ce primordial pour une recherche des liens entre cette méthode et le travail, celui-ci n'étant jamais mobilisé de façon abstraite, mais, au contraire, dans des contextes productifs, à savoir des types de produits à réaliser, porteurs de contraintes spécifiques (dans la gestion du temps, dans la présence ou non du client dans les opérations de production, dans la matérialité des actes à effectuer, dans les critères de qualité...), des technologies, des formes de travail (plutôt individuelles ou, au contraire, collectives, reconnues comme qualifiées ou non, etc.). L'extension à une variété de configurations productives est à regarder de près. De fait, l'élaboration première de la méthode et des outils s'est faite à partir de cadres productifs très précis et pour des types de mobilisation du travail qui ne sont pas obligatoirement universels. C'est dans cet espace qu'a pu être mobilisée, non une théorie du travail en général, mais une théorie gestionnaire se donnant implicitement un type de travail. Si la méthode et les outils sont destinés à aller s'appliquer dans d'autres contextes, il y a lieu d'en tenir compte. La variété de ces configurations productives pourra même conduire à s'interroger: est-ce toujours du lean? Mais, aussi, qu'est-ce qui, au fond, est réellement « lean »?

Le lean n'est pas une substance. Il n'y a pas d'identité stable et «véridique » du lean. Par exemple, il est parfois associé à «Six Sigma», dans des manuels sur «lean six sigma » qui laisseront entendre qu'il s'agit sans aucun doute possible de la recherche des mêmes objectifs; parfois, au contraire, il est considéré comme de nature fondamentalement différente. Ce n'est pas non plus une liste consensuelle d'outils. Les auteurs - prescripteurs ou observateurs - en font des assortiments jamais identiques, comme l'a montré Pettersen (2009) (Tableau 1).

\begin{tabular}{|c|c|c|c|c|c|c|c|c|c|}
\hline & $\begin{array}{c}\text { Womack \& } \\
\text { Jones } \\
\text { (\& Roos) }\end{array}$ & Liker & Bicheno & Dennis & Feld & Ohno & Monden & Schonberger & Shingo \\
\hline Kaizen/Continuous improvement & $\mathrm{x}$ & $\mathrm{x}$ & $\mathrm{x}$ & $\mathrm{x}$ & $\mathrm{x}$ & $\mathrm{x}$ & $\mathrm{x}$ & $\mathrm{x}$ & $x$ \\
\hline Setup time reduction & $\mathrm{x}$ & $\mathrm{x}$ & $\mathrm{x}$ & $\mathrm{x}$ & $\mathrm{x}$ & $\mathrm{x}$ & $\mathrm{x}$ & $\mathrm{x}$ & $\mathrm{x}$ \\
\hline Just in time production & $\mathrm{x}$ & $\mathrm{x}$ & & $\mathrm{x}$ & $\mathrm{x}$ & $x$ & $\mathrm{x}$ & $\mathrm{x}$ & $\mathrm{x}$ \\
\hline Kanban/Pull system & $\mathrm{x}$ & $\mathrm{x}$ & $\mathrm{x}$ & $\mathrm{x}$ & $\mathrm{x}$ & $\mathrm{x}$ & $\mathrm{x}$ & $\mathrm{x}$ & \\
\hline Poka yoke & & $\mathrm{x}$ & $\mathrm{x}$ & $\mathrm{x}$ & $\mathrm{x}$ & $\mathrm{x}$ & $\mathrm{x}$ & $\mathrm{x}$ & $\mathrm{x}$ \\
\hline Production leveling (Heijunka) & $\mathrm{x}$ & $\mathrm{x}$ & $\mathrm{x}$ & $\mathrm{x}$ & $\mathrm{x}$ & $\mathrm{x}$ & $\mathrm{x}$ & & $\mathrm{x}$ \\
\hline Standardized work & & $\mathrm{x}$ & $\mathrm{x}$ & $\mathrm{x}$ & $\mathrm{x}$ & $\mathrm{x}$ & $\mathrm{x}$ & $\mathrm{x}$ & $\mathrm{x}$ \\
\hline Visual control and management & & $\mathrm{x}$ & $\mathrm{x}$ & $\mathrm{x}$ & $\mathrm{x}$ & $\mathrm{x}$ & $\mathrm{x}$ & $\mathrm{x}$ & $\mathrm{x}$ \\
\hline 5S/Housekeeping & $\mathrm{x}$ & $\mathrm{x}$ & $\mathrm{x}$ & $\mathrm{x}$ & $(\mathrm{X})$ & $\mathrm{x}$ & $\mathrm{x}$ & $\mathrm{x}$ & \\
\hline Andon & $\mathrm{x}$ & $\mathrm{x}$ & & & $\mathrm{x}$ & $\mathrm{x}$ & $\mathrm{x}$ & $\mathrm{x}$ & $\mathrm{x}$ \\
\hline Small lot production & & $x$ & $x$ & & $\mathrm{x}$ & $\mathrm{x}$ & $\mathrm{x}$ & $x$ & $\mathrm{x}$ \\
\hline Time/Work studies & $\mathrm{x}$ & $\mathrm{x}$ & $\mathrm{x}$ & $\mathrm{x}$ & $\mathrm{x}$ & $\mathrm{x}$ & $\mathrm{x}$ & & \\
\hline Waste elimination & $\mathrm{x}$ & $\mathrm{x}$ & $\mathrm{x}$ & $\mathrm{x}$ & & $\mathrm{x}$ & & $\mathrm{x}$ & $\mathrm{x}$ \\
\hline Inventory reduction & $\mathrm{x}$ & $\mathrm{x}$ & & $\mathrm{x}$ & & $\mathrm{x}$ & $\mathrm{x}$ & $\mathrm{x}$ & $\mathrm{x}$ \\
\hline Supplier involvement & $\mathrm{x}$ & $\mathrm{x}$ & $\mathrm{x}$ & $\mathrm{x}$ & $\mathrm{x}$ & & $\mathrm{x}$ & & \\
\hline Takted Production & & $\mathrm{x}$ & $\mathrm{x}$ & $\mathrm{x}$ & $\mathrm{x}$ & & $\mathrm{x}$ & & $\mathrm{x}$ \\
\hline TPM/Preventive mainenance & & $\mathrm{x}$ & $x$ & $\mathrm{x}$ & $\mathrm{x}$ & $x$ & & $x$ & \\
\hline Autonomation (Jidoka) & & $\mathrm{x}$ & & $\mathrm{x}$ & & & $\mathrm{x}$ & $\mathrm{x}$ & $\mathrm{x}$ \\
\hline Statistical quality control (SQC) & $\mathrm{x}$ & & $\mathrm{x}$ & NO! & $\mathrm{x}$ & & $\mathrm{x}$ & $\mathrm{x}$ & \\
\hline Teamwork & $\mathrm{x}$ & $\mathrm{x}$ & & $\mathrm{x}$ & $\mathrm{x}$ & $\mathrm{x}$ & & & \\
\hline Work force reduction & & & & $\mathrm{x}$ & & $\mathrm{x}$ & $\mathrm{x}$ & $\mathrm{x}$ & $\mathrm{x}$ \\
\hline $100 \%$ inspection & & $\mathrm{x}$ & & $\mathrm{x}$ & & & & $\mathrm{x}$ & $\mathrm{x}$ \\
\hline Layout adjustments & & & & $\mathrm{x}$ & & & $\mathrm{x}$ & $\mathrm{x}$ & $\mathrm{x}$ \\
\hline Policy deployment (Hoshin kanri) & $\mathrm{x}$ & $\mathrm{x}$ & $\mathrm{x}$ & $\mathrm{x}$ & & & & & \\
\hline Improvement circles & & $\mathrm{x}$ & & $x$ & & & $\mathrm{x}$ & $x$ & \\
\hline Root cause analysis ( 5 why) & $\mathrm{x}$ & $\mathrm{x}$ & $\mathrm{x}$ & & & $\mathrm{x}$ & & & \\
\hline Value stream mapping/flowcharting & $x$ & $\mathrm{x}$ & $\mathrm{x}$ & $\mathrm{x}$ & & & & & \\
\hline Education/Cross training (OJT) & & $\mathrm{x}$ & & & $\mathrm{x}$ & & & $\mathrm{x}$ & \\
\hline Employee involvement & $\mathrm{x}$ & $x$ & & $\mathrm{x}$ & & & $(\mathrm{X})$ & & \\
\hline Lead time reduction & & $\mathrm{x}$ & & $\mathrm{x}$ & & & $x$ & & \\
\hline Multi manning & $(\mathrm{X})$ & & & & & $\mathrm{x}$ & $\mathrm{x}$ & & $\mathrm{x}$ \\
\hline Process synchronization & & $\mathrm{x}$ & & & & & & $\mathrm{x}$ & $\mathrm{x}$ \\
\hline Cellular manufacturing & & & $\mathrm{x}$ & & $\mathrm{x}$ & & $(\mathrm{X})$ & & \\
\hline Goal & $\begin{array}{l}\text { make } \\
\text { products with } \\
\text { fewer defects } \\
\text { to precise } \\
\text { customer } \\
\text { desires }\end{array}$ & $\begin{array}{c}\text { One-piece } \\
\text { flow }\end{array}$ & $\begin{array}{c}\text { Reduce } \\
\text { waste and } \\
\text { improve } \\
\text { value }\end{array}$ & $\begin{array}{l}\text { Customer } \\
\text { focus (high } \\
\text { quality, low } \\
\text { cost, short } \\
\text { time) }\end{array}$ & $\begin{array}{c}\text { Robust } \\
\text { production } \\
\text { operation }\end{array}$ & $\begin{array}{c}\text { Cost } \\
\text { reduction }\end{array}$ & $\begin{array}{l}\text { Eliminate } \\
\text { waste and } \\
\text { reduce costs }\end{array}$ & $\begin{array}{l}\text { Improve } \\
\text { quality and } \\
\text { productivity }\end{array}$ & $\begin{array}{l}\text { Cost } \\
\text { reduction } \\
\text { through } \\
\text { waste } \\
\text { elimination }\end{array}$ \\
\hline
\end{tabular}

Tableau 1 : Les outils du lean selon divers auteurs

Table 1: Lean tools according to various authors

Source : Pertersen (2009) 
Cela ne joue pas qu'à la marge, mais sur des outils parfois jugés décisifs : ainsi, le Takt time est-il une notion pivot pour un observateur comme Shimizu, alors que les inventeurs de l'idée d'une lean production, Womack, Jones et Roos, n'y font pas allusion (Shimizu, 1999 ; Womack, Jones, \& Roos, 1990). En somme, le lean n'est pas une substance, mais s'observe plutôt autour de modes d'existence pluriels.

\section{2.- Lean production, lean management, lean}

Le vocabulaire n'est pas plus unifié. Système de production Toyota (SPT), lean production, lean manufacturing, lean management, lean, pour ne pas parler des lean logistics, lean software, lean and green et ainsi de suite. On parlait aussi, initialement, chez les observateurs, de toyotisme ou de ohnisme. Qui plus est, chaque entreprise adaptant à ses particularités la méthode empruntée à Toyota en fait un «système de production » dans lequel on substitue à Toyota le nom de la firme. Au bout du compte, on est devant des variations de vocabulaire difficiles à organiser, et cependant qui comptent pour une réflexion sur les conséquences pour l'activité. Conventionnellement, je proposerai d'appeler lean production le modèle pensé à partir de l'industrie automobile, lean manufacturing un modèle prétendant à plus de généralité au sein de l'industrie et lean management une mode managériale ayant l'ambition de couvrir encore plus large.

On dessine ainsi une trajectoire dont le point d'origine est le système de production Toyota, tel qu'en rend compte, par exemple, son concepteur Ohno, dans son ouvrage en 1978 (Ohno, 1978). Lorsque, courant des années 1980, on invente l'expression de lean production pour désigner ce modèle de production et l'ériger en modèle, on est censé se situer sur la même ligne, mais c'est également l'ouverture d'une trajectoire autonome pour un modèle ayant prétention à des formes de généralité, au-delà du cas particulier de Toyota. L'ouvrage de référence est celui que publient Womack, Jones et Roos, en 1990 (pour une histoire de lean production de Ohno à la lean production et à ses premières applications au-delà des frontières japonaises, voir Holweg, 2006). À partir du moment où la lean production a fait concevoir la question du modèle, une question est celle du domaine de validité et d'extension de ce modèle. Dès lors qu'il s'agit de défendre que ce ne sont pas seulement les constructeurs automobiles - auxquels s'étaient limités Womack et ses co-auteurs - qui sont concernés, mais l'ensemble des processus manufacturiers, on franchit une étape supplémentaire, qui se ressent dans le besoin de faire évoluer le vocabulaire et de parler d'un lean manufacturing, lequel ouvre, à son tour, sa propre trajectoire. Dans un stade supplémentaire de l'extension, il s'agira de dire qu'on a affaire à un modèle de management valant de façon si générale qu'il peut se défendre en dehors de toute référence aux seules productions manufacturières, et l'on parlera alors de lean management. C'est encore une trajectoire qui va vivre sa vie propre. À chacune de ces étapes, il s'agit de plus en plus de se détacher de la référence à la configuration productive de série typique de l'industrie automobile et de cesser de donner à voir le travail de l'opérateur sur chaîne de montage.

Quand on parle du lean (le français permet de voir que, passant du féminin au masculin, on a, en fait, délaissé la référence à la lean production pour lui préférer celle du lean management ou manufacturing), on est face aux conceptions les plus généralisantes et donc aussi celles qui renvoient le moins explicitement vers les activités de travail concernées par les premiers stades de l'élaboration du modèle. On est donc aussi face à des renvois éventuellement plus flous aux implications concrètes pour le travail. Enfin, quand l'expression est «être lean », il s'agit de l'idée d'être dans l'état d'esprit d'appliquer les principes du lean management et il n'est plus forcément question de gestion de production de façon précise. Dans l'affaire, en tout cas et comme tend à le montrer le tableau 2, ces appellations les plus englobantes ont supplanté celle de lean production, qui paraît du même coup plus restrictive. En se limitant à un quotidien du monde économique comme Les Échos, on voit qu'une requête sur les termes «lean » et «production » fait ressortir une mention de la lean production à la fin des années 1990 pour disparaître quelque temps avant que ne 
resurgissent de nouvelles expressions. Au milieu des années 2000, c'est le lean manufacturing et le lean management qui réimposent le sujet.

\begin{tabular}{|l|c|c|c|c|c|c|c|}
\hline & $\begin{array}{c}\text { Lean } \\
\text { produc- } \\
\text { tion }\end{array}$ & $\begin{array}{c}\text { Lean } \\
\text { manu- } \\
\text { facturing }\end{array}$ & $\begin{array}{c}\text { Lean } \\
\text { mana- } \\
\text { gement }\end{array}$ & Lean & $\begin{array}{c}\text { Lean } \\
\text { logistics }\end{array}$ & $\begin{array}{c}\text { Lean } \\
\text { and } \\
\text { green }\end{array}$ & TOTAL \\
\hline 1997 & 1 & & & & & & 1 \\
\hline 1998 & 3 & & & & & & 3 \\
\hline 1999 & 2 & & & & & & 2 \\
\hline 2000 & 2 & & & & & & 2 \\
\hline 2001 & 0 & & & & & & 0 \\
\hline 2002 & 0 & & & & & & 0 \\
\hline 2003 & & 2 & & & & & 2 \\
\hline 2004 & 1 & & & & & & 1 \\
\hline 2005 & & 2 & 1 & 1 & & & 4 \\
\hline 2006 & & 1 & 2 & & & & 3 \\
\hline 2007 & 2 & 1 & & 1 & & & 4 \\
\hline 2008 & 1 & 2 & 1 & & & & 4 \\
\hline 2009 & 2 & 1 & 4 & 1 & 2 & & 9 \\
\hline 2010 & 1 & 3 & 4 & 1 & & & 9 \\
\hline 2011 & & 6 & 1 & 1 & & & \\
\hline
\end{tabular}

Tableau 2 : La résurgence du lean : occurrences de «lean » et «production » dans Les Échos

Table 2: The resurgence of lean: occurrences of "lean" and "production" in Les Échos

Source: Données collectées sur Factiva ${ }^{\circledR}$. La requête a été formulée «lean and production ». Il ne s'agit donc pas de la totalité des articles faisant mention du lean ou du lean manufacturing ou du lean management. La requête incluant le mot «production », elle a probablement conduit à surreprésenter les articles concernant l'industrie et le lean manufacturing. Les articles mentionnant «lean» et «production», mais sur des sujets étrangers ont été exclus des comptages.

\section{2.- La lean production et ses principes : quelle représentation du travail ?}

Si l'on décide d'enquêter sur la théorie de l'activité sous-jacente au lean, il semble indispensable de partir de la théorie de l'activité présente dans la doctrine de la lean production ou du SPT, avec des références comme Ohno et Womack et ses coauteurs. On est ici au plus près de l'invention des principes et des outils et c'est, du reste, là que l'on trouvera l'idée du travail la plus explicite. Il faudra néanmoins se demander ensuite quelle extension au « lean » est possible.

\section{1.- Un modèle d'activité difficile à trouver}

Le modèle d'activité sous-jacent au lean ne se révèle pas de façon aussi immédiate qu'on pourrait l'espérer. Il faut quelque peu aller le chercher. Tout d'abord, une difficulté tient au fait qu'il existe une variété des textes «fondateurs » : au premier rang de ceux-ci, les écrits des créateurs du SPT, en particulier son grand artisan, Taiichi Ohno. Ingénieur ayant fait toute sa carrière chez le constructeur japonais jusqu'à en devenir le vice-président, il élaborera la méthode par tâtonnement et par inventions successives, avant d'en rendre compte dans un ouvrage en 1978, traduit en français onze ans plus tard (Ohno, 1978). Mais aussi les écrits assez militants de chercheurs du Massachusetts Institute of Technology, qui édifieront l'idée d'une lean production et la populariseront dans l'ouvrage, devenu un best seller, The Machine that Changed the World (Womack, et al., 1990), avec une traduction française en 1992.

Par ailleurs, se pose inévitablement la question de savoir la portée qu'on donne à ce qu'on trouvera dans de tels textes à partir du moment où on a rapidement su qu'un écart pouvait exister entre ce qui était décrit et érigé en modèle par ces textes et les applications en 
contexte, porteuses de variations. Au début des années 1990, c'était un thème récurrent des analyses portant sur le toyotisme et sa diffusion de par le monde et, plus spécifiquement, sur l'étude des «transplants» (Fairris \& Tohyama, 2002 ; Rinehart, Huxley, \& Robertson, 1994 ; Stewart, Durand, Lewchuk, Yates, \& Danford, 2006 ; pour une application du modèle de Karasek aux personnels d'un transplant en 1999, voir Schouteten et Benders, 2004).

Toute une production d'analyses a pu exister à cette période qui n'a pas nécessairement aidé à faire émerger le modèle d'activité sous-jacent à la lean production. Ces analyses trouvaient prioritairement intérêt à débattre de ses principes généraux et, éventuellement, de résistances nationales à son application pure et simple, davantage qu'à étudier de façon serrée les conséquences pour l'activité ${ }^{1}$. La caractéristique de cette génération de textes est d'être dominés par les préceptes autant ou plus que par les outils. François Dalle le relève à propos de l'ouvrage d'Ohno lorsqu'il écrit dans la préface à la traduction française : "Ce livre n'est donc pas un livre de recettes. C'est un livre de préceptes.» (p. 6). Dans les années 1980 et 1990, le débat sur ce que la méthode Toyota fait au travail est également instruit au niveau des grands principes. Lorsque des outils sont mentionnés, c'est en petit nombre - notamment le kaizen -, et ils sont traités moins pour eux-mêmes que, en quelque sorte, comme des véhicules des principes (la mobilisation de l'initiative des opérateurs). Une grande partie du débat s'organise autour de la réalité ou non du principe du travail en équipe (qui n'apparaîtra guère, au contraire, aujourd'hui, comme une notion décisive du lean) (pour un exemple plus récent, voir Vidal, 2007). L'activité de travail sera, en tout cas, relativement peu décrite.

Enfin, dernier point, essentiel, ni les écrits d'Ohno, ni ceux de Womack et ses collègues ne sont spécialement «tirés » par une théorie du travail et ne cherchent à l'être. Ce ne sont pas les pendants contemporains des écrits de Taylor. Ce dernier offre, à son époque, une pensée extrêmement construite de la façon dont le travail prend place dans une activité productive dirigée et de sa contribution spécifique au rendement économique. C'est une pensée qui s'inscrit dans l'histoire plus générale de la conceptualisation du travail par les ingénieurs, qui ont été très actifs à élaborer une définition («physicaliste ») du travail (Vatin, 2003). Le lean hérite de cette pensée d'ingénieurs sans entreprendre de s'y singulariser. Ohno est préoccupé par l'ingénierie de production sans que celle-ci, dans la façon dont il la relate, n'ait le travail des opérateurs comme obsession. Il passe par là, il l'évoque, ce qu'il conçoit concerne très directement le travail, mais sans donner l'impression qu'il aurait appréhendé le travail comme devant donner lieu à une théorie pour les besoins mêmes de son œuvre d'invention. La présentation qu'il fait de sa propre activité d'invention donne beaucoup plus de place aux problèmes pratiques à résoudre concernant tous types de paramètres d'une ingénierie de la production. Womack et ses collègues ne seront pas non plus porteurs d'une théorie spécifique du travail. Ainsi, ces textes ne livrent pas explicitement un modèle de l'activité de travail.

\section{2.- La base, un principe de combat quotidien contre les gaspillages}

Chez ces auteurs fondateurs, la réflexion se concentre sur l'industrie automobile et porte la marque de la référence à ce type d'activité productive qu'est la production industrielle en

\footnotetext{
Par ailleurs, un témoignage comme celui de Kamata, relatant ce que veut dire être ouvrier chez Toyota, ne peut être apprécié - contrairement à ce que font Schouteten et Benders (2004) - comme documentant les conditions réelles de mobilisation de l'activité dans le cadre des principes spécifiquement toyotiens (Kamata, 1973). Le témoignage de ce journaliste s'est fondé sur une expérience de travail en 1972. L'ouvrage, qui paraît en 1973, renseigne essentiellement sur ce que veut dire travailler à la chaîne dans l'industrie automobile à cette époque. Aucun des principes ni des outils caractéristiques du SPT n'est mentionné, en dehors d'une vague allusion à ce qui était alors les $4 \mathrm{~S}$ (aujourd'hui au nombre de cinq). Ressort davantage le contexte de croissance exponentielle à laquelle était soumis le constructeur à cette époque, qui l'amenait à privilégier une logique de débit et d'intensification du travail, faute de faire évoluer dans les mêmes proportions l'outil de production et l'effectif employé.
} 
séries standardisées. Plus spécifiquement, c'est même l'assemblage qui est la référence la plus fréquente. Autant dire des activités productives où il y a de forts enjeux de planification de la production, dans un système mobilisant des milliers de composants. Même avec de plus courtes séries, sur quoi le modèle Toyota se distingue, la préoccupation est celle du débit et de la productivité correspondante (Veltz \& Zarifian, 1993). En toile de fond se trouve un certain consensus, en gestion de production, au milieu du $X^{\mathrm{e}}$ siècle, vis-à-vis de la supériorité du modèle productif de Ford : les temps de réglage des équipements entre lots de fabrication étant longs et difficilement compressibles, il faut rechercher l'allongement de ces lots de production, quitte à constituer des stocks intermédiaires. Décisif pour la genèse du SPT, au contraire, est le fait que cette obligation de constituer des lots de grande taille représente une contrainte qui ne concorde pas avec tous les marchés, notamment le marché japonais de l'automobile des années 1940 et 1950, de taille modeste. Ce sera le point de départ de la réflexion d'Ohno, amené à devoir trouver de quoi contourner cet obstacle pour cependant augmenter la productivité, ce qui le conduira à considérer qu'il s'agit là, par ailleurs, d'un mode de fonctionnement ayant pour défaut d'engendrer des gaspillages.

Le SPT prévoit donc une mise en fabrication de petits lots, avec l'obsession d'éviter les gaspillages. Au fond, c'est une méthode d'amélioration de la productivité du capital circulant (F. Dalle, préface à Ohno, 1978, p. 5). Womack, Jones et Roos défendent, dans une perspective quasi évolutionniste, que c'est une tendance de l'histoire que ce déplacement de la production de masse vers la production « au plus juste » (telle est la traduction française courante de «lean ») dans l'industrie automobile. Ils le tirent d'une enquête menée à partir de 1986 dans les usines de montage chez les constructeurs et sous-traitants (à nouveau de l'assemblage pur).

Ils écrivent, à propos d'une visite faite dans une usine de General Motors en 1986 :

«Puis nous inspectâmes la chaîne elle-même. Près de chaque poste de travail se trouvent des piles (...) de pièces. Eparpillés tout autour, des cartons vides et d'autres emballages éventrés jonchaient le sol. Sur la chaîne, le travail était de toute évidence mal réparti, certains ouvriers courant dans tous les sens pour garder le rythme général tandis que d'autres trouvaient le temps de fumer ou même de lire un journal (...)

En bout de chaîne, nous découvrîmes ce qui est probablement le traitement le plus caractéristique de la production de masse : un énorme espace consacré aux véhicules défectueux. Tous ces véhicules nécessitaient une réparation avec l'expédition (...).

Sur notre chemin de retour (...) nous remarquâmes deux dernières images caractéristiques de la production de masse : les énormes quantités de carrosseries vides attendant d'entamer leur pèlerinage sur la chaîne après leur passage dans les cabines de peinture et les énormes stocks de pièces, parfois encore entassés dans leurs wagons en provenance des fournisseurs. » (Womack et al., 1990, p. 94).

Chez Toyota, au contraire, « presque personne ne se trouvait dans les dégagements. » (ibid., p. 95).

L'usine au plus juste présente ainsi, pour ces auteurs, deux caractéristiques fondamentales : «elle délègue le maximum de tâches et de responsabilités aux intervenants apportant effectivement de la valeur ajoutée au produit - les ouvriers sur chaîne » (et non pas, par exemple, les retoucheurs); "elle fonctionne sur un système de détection et de solution précoce des défauts.» (ibid., p. 117). "Cela suppose un travail d'équipe sans faille et un système d'information simple et global permettant à quiconque, quel que soit son échelon, de réagir rapidement aux demandes et de connaître parfaitement la situation de l'usine » (ibid.). C'est ici que se trouve affirmée par les promoteurs de la lean production cette idée qui était peut-être moins centralement affichée chez Ohno, celle de la montée en responsabilité des opérateurs. C'est aussi le sujet sur lequel le débat a porté durant les années 1990 : réalité ou non de ce principe.

Chaque poste de travail prélève sur celui qui le précède les composants qui lui sont immédiatement et juste nécessaires - en informant ce dernier des quantités qu'il doit 
refabriquer (grâce aux kanbans, à la fois bons de prélèvement, ordres de transfert et ordres de fabrication). Le kanban implique que le poste de travail ne peut pas organiser sa journée à partir d'ordres de fabrication donnés par le planning mais doit s'adapter aux commandes du poste de travail aval. Le point sera évidemment crucial pour l'activité de travail.

Pour ne pas imposer au poste de travail amont une perturbation trop déstabilisante de ses ordres de fabrication (par exemple, une demande de quantités exceptionnellement importantes), il faut niveler le plan de production en faisant de très courtes séries, ce qui suppose de constamment réduire les temps de changement d'outils d'un lot à l'autre ${ }^{2}$, avec là encore une implication assez évidente pour l'activité de travail qui, dans la disparition ou réduction de ces temps considérés comme improductifs, voit aussi s'amenuiser les moments de respiration, les temps où les opérateurs peuvent récupérer. Il faut, par ailleurs, produire sans défaut (le kanban ne circule que pour des pièces pouvant être garanties absolument correctes). Un défaut sur le moindre composant compromet le plan de production. Dans le modèle américain, c'est-à-dire fordien, pour éviter d'arrêter le flux productif, on avait pris l'habitude d'avoir des stocks, ce qui, du côté Toyota, s'interprète comme une solution pesant sur la rentabilité et, par ailleurs, masquant les anomalies et retardant leur correction. Le point n'est pas secondaire, les principes inventés chez Toyota portant à considérer que de petits détails, même d'une visibilité et d'une portée apparente relatives, doivent être érigés en problème digne d'intérêt. Dans la conception élaborée par Ohno, il n'y a pas de grands et nobles problèmes d'ingénieurs et de calcul économique, à côté desquels d'autres, constatés dans le quotidien du fonctionnement des ateliers, seraient de faible portée. Il y a, au contraire, une importance déclarée de ce qui saute aux yeux quand on se place au niveau de ce quotidien et des acteurs qui y interviennent, comme la maîtrise ou les opérateurs. Cela également dénote une représentation, sinon du travail, du moins des diverses échelles des problèmes de production et cela peut valoriser l'activité. Une conséquence traduite dans les principes et les outils sera que, dans le SPT, les machines sont programmées pour - et les hommes sont habilités à - s'auto-arrêter en cas d'anomalie (poka yoke), ce qui est une traduction de la montée en responsabilité dans le travail que des auteurs comme Womack et ses collègues ont mis en avant.

\section{3.- La face technique du lean : ce que l'on sait en creux de l'activité}

Ce sont donc les effets en chaîne de modifications de procédés dans l'ingénierie de production qui conduisent à la conception de ce nouvel ensemble, lequel, dès lors, fait système et peut être présenté par Ohno à la fin des années 1970 dans une certaine cohérence d'ensemble. Des principes ont fini par être mis à jour et des dispositifs ont été élaborés, ceux que les manuels et les consultants présenteront par la suite dans la boîte à outils comme allant «nécessairement» avec un «esprit» lean. C'est à la confluence de l'intention générale du lean et de quelques-uns de ces dispositifs qu'il faut aller chercher les implications pour l'activité de travail.

L'intention générale du lean, en résumé, est donc que « la production au plus juste consiste à réduire continuellement le temps nécessaire à la transformation d'une matière première en produit fini, en éliminant les gaspillages. » Et cela « en se calant sur le délai final (Lasnier, 2007, p. 99). Cela passe par une série de dispositifs. Le premier d'entre eux à évoquer est sans doute le takt time.

2 D'où l'outil qui sera, plus tard, valorisé sous le nom de Single minute exchange of die (SMED). 


\section{1.- Le takt time et ses effets pour l'activité de travail}

Empruntons à Lasnier (2007) l'illustration suivante.

«Soit une ligne de production travaillant en 2 équipes à raison de 7 heures par jour. Le temps d'ouverture de la ligne est donc de $2 \times 7 \times 60 \mathrm{mn}=840$ minutes ou 50400 secondes. La demande de la clientèle est de 4200 pièces par jour, le takt time TT est de 50 400/4 200 $=12$ secondes par pièce.

Si l'on veut supprimer les en-cours et autres problèmes dans la ligne, les différentes opérations dans le flux doivent adopter le même rythme de 12 secondes. Il s'agit ensuite de déterminer le nombre de postes et d'adapter en fonction des cadences à chaque poste un effectif d'opérateurs. (...) Un premier calcul va permettre d'évaluer le nombre optimal de postes en se basant sur la cadence moyenne tous produits confondus. » (Lasnier, 2007, p. 101).

Le nombre optimal de postes est le rapport entre temps opératoire moyen par produit à partir des gammes et le takt time. Si le temps défini par les gammes est de 96 secondes, alors le nombre d'opérateurs est égal à 96/12, soit 8 .

Il faut, dès lors, répartir les opérateurs en fonction des temps opératoires à chaque poste, selon le principe illustré par le tableau 3.

\begin{tabular}{|l|c|c|c|c|c|c|}
\hline & $\begin{array}{c}\text { Opé- } \\
\text { ration 1 }\end{array}$ & $\begin{array}{c}\text { Opé- } \\
\text { ration 2 }\end{array}$ & $\begin{array}{c}\text { Opé- } \\
\text { ration 3 }\end{array}$ & $\begin{array}{c}\text { Opé- } \\
\text { ration 4 }\end{array}$ & $\begin{array}{c}\text { Opé- } \\
\text { ration 5 }\end{array}$ & TOTAL \\
\hline Temps par pièce & $24 \mathrm{~s}$ & $6 \mathrm{~s}$ & $6 \mathrm{~s}$ & $48 \mathrm{~s}$ & $12 \mathrm{~s}$ & $96 \mathrm{~s}$ \\
\hline $\begin{array}{l}\text { Nombre } \\
\text { d'opérateurs }\end{array}$ & 2 & & 1 & 4 & 1 & 8 opérateurs \\
\hline
\end{tabular}

Tableau 3 : Répartition de l'effectif en fonction de la valeur du takt time

Table 3: Employee distribution as a function of takt time value

Source : Lasnier (2007), p. 101.

Shimizu montre, quant à lui, comment on réorganisera les tâches pour homogénéiser les temps d'occupation des opérateurs et, si possible, gagner un poste au passage.

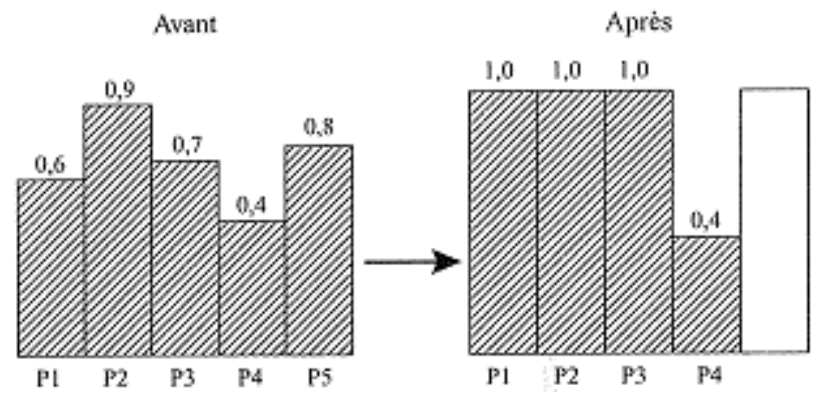

Figure 1 : Réorganisation des tâches d'une ligne pour un takt time égal à 1 minute et des postes P1 à P5.

Figure 1: Reorganisation of line tasks for a takt time of 1 minute and stations P1 to P5

Source : Shimizu (1999), p. 45.

C'est dans ce cadre que va trouver son sens la formule devenue célèbre de l'organisation en $\mathrm{U}$ de la ligne de production, permettant d'organiser la présence des opérateurs sur diverses opérations dont le total répond à la valeur uniforme attendue. 


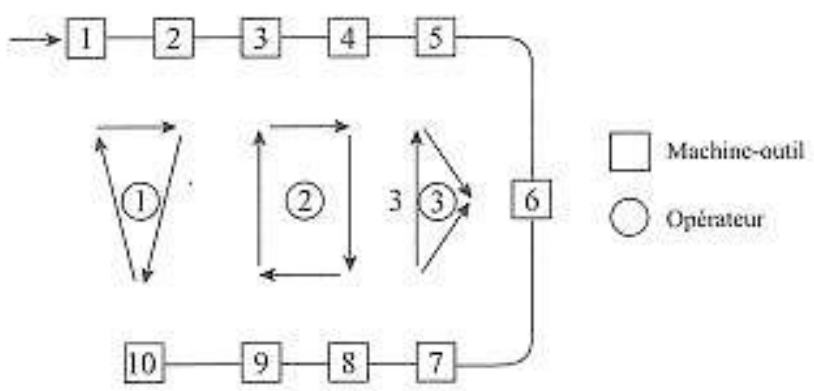

Figure 2 : La ligne en $\mathrm{U}$.

Figure 2: The U-line.

Source : Shimizu (1999), p. 46.

On notera le rôle central des gammes ou temps opératoires. Ohno $(1978$, p. 35) définit le standard opératoire par le cycle opératoire («temps alloué à l'exécution d'une pièce »), gamme, stock-outil standard. On est bien ici dans la droite ligne de l'organisation industrielle contestant aux métiers le contrôle de la production et des procédés (Ohno, 1978, p. 24). Au début des années 1990, Coriat en livrait une interprétation selon laquelle on avait, certes, affaire à un processus d'intensification du travail, mais qui «au lieu de procéder par parcellisation et micro-temps imposés » comme le taylorisme-fordisme, "procède ici par déspécialisation et "temps partagé" »(Coriat, 1991, p. 45). On peut tout autant y voir un travail sous contrainte de temps, celle des «organisations au plus juste» dont les caractéristiques sont résumées par Valeyre (2006) : lourdes contraintes de rythme, tâches répétitives et monotones. Le principe est bien l'optimisation de l'utilisation du temps des opérateurs. Morvan, François et Bourgeois (2008) montrent, à partir d'une étude de cas, que l'activité se trouve considérablement compacifiée par le modèle de l'opérateur tournant associé à la ligne en $\mathrm{U}$, amené à se déplacer sur la ligne, avec des implications coûteuses sur plusieurs plans :

«[Les] déplacements sont effectués dans un espace limité, sous contrainte temporelle, en tenant compte de la position dans l'espace des collègues pour garder la bonne distance, le bon rythme... (...) L'appareil locomoteur est fortement sollicité, de manière statique et dynamique. C'est un mode d'organisation qui demande un effort physique supplémentaire pour l'opérateur, par rapport aux postes fixes assis.

(...) Se coordonner au sein de l'équipe de la ligne en U est aussi un travail. (...) dans les configurations en opérateurs tournants, la synchronisation entre les activités des uns et des autres dans la cellule en $\mathrm{U}$ doit être ajustée de manière fine et réciproque par chaque opérateur en fonction du contexte. (...) Pour assurer la fluidité du processus, il faut donc savoir jouer collectivement sur les temps de déplacement et sur les temps d'activités sur les machines. Il faut se synchroniser les uns les autres pour produire, comme pour éviter les collisions. Il faut anticiper les aléas et la gêne des autres opérateurs. » (Morvan, François et Bourgeois, 2008, pp. 2-3).

\section{2.- Du taux de rendement synthétique à la réimplantation d'atelier pour diminuer les déplacements}

Le lean invite à s'attaquer aux gaspillages indépendamment du degré de gravité qui serait $a$ priori le leur, ce qui semble dessiner un ensemble parfaitement hétéroclite. La notion de taux de rendement synthétique (TRS) ordonne cependant tout cela en dégageant les grandes catégories de gaspillages - ou mudas - à chasser. Le TRS traduit le rendement effectif d'installations de production compte tenu de tout ce qui entame leur fonctionnement théorique. Son calcul oblige à opérer les décompositions suivantes (Lasnier, 2007, pp. 101102).

Partant du temps d'ouverture de l'installation de production, on sait que des arrêts tout à fait 
planifiés (par exemple pour maintenance) vont limiter celui-ci. La différence constitue le temps requis. À son tour, celui-ci est diminué d'autres arrêts, ceux dus aux pannes, qui n'avaient pu être prévus, et ceux liés aux changements de série, donnant lieu au temps de fonctionnement brut. Lui-même est, dans les faits, réduit par les temps perdus à la suite du non-respect des cadences, celles des machines ou celles des hommes, ce qui correspond au temps de fonctionnement net. Cela traduit la performance productive réelle. Mais, par ailleurs, le lean est attentif au fait que cette performance perd de son sens si elle sert à fabriquer des produits présentant des défauts. On dégage donc le temps utile en décomptant du temps de fonctionnement net le temps perdu à de telles fabrications. Le TRS sera le rapport entre le temps utile et le temps d'ouverture.

Cette décomposition permet d'identifier les grands champs d'action auxquels il conviendra de se rendre attentif pour réduire tout ce qui est improductif et se traduit par une diminution du TRS. Maximiser le TRS, c'est maximiser le rapport temps utile/ temps requis en agissant sur les pannes, les changements de série, les micro-défaillances, les problèmes de cadence, les problèmes de qualité.

C'est alors de façon tout à fait instrumentée, par de la mesure et non par une pure croyance dans les vertus d'une mobilisation des esprits en faveur de la performance, que se comprend l'idée de démarches d'amélioration continue, le fameux kaizen. Ces démarches se conçoivent comme devant se faire avec les acteurs concernés, que l'on trouvera au niveau de la fabrication, mais aussi de la qualité, de la maintenance, des méthodes, de la logistique, des achats, cette coopération devant viser à réduire les pertes à tous les niveaux visibles dans la décomposition et à optimiser la disponibilité (temps d'utilisation), la performance (respect des cadences) et la qualité (zéro défaut). Par exemple, la cadence est donnée par le takt time. Elle peut être améliorée par le 5S (optimisation de l'ordre et du rangement en clarifiant les emplacements où l'on approvisionne le poste).

Le taux de rendement synthétique est le ratio qui rend très perceptible l'obsession à l'égard des actes non directement productifs. Il oriente la réflexion vers l'objectivation de ce qui mobilise de l'activité sans débouché direct apparent. La notion est porteuse de toute l'ambivalence du lean : l'attention à tout ce qui n'est pas d'un rendement réel fait tout aussi bien découvrir des possibilités de soulager l'activité humaine de gestes ou d'actes qui pourraient être évités que générer une compacification du temps qui met les opérateurs dans une tension sans relâchement vers la production.

Avec une telle notion prend sens le diagnostic porté par des opérateurs de l'automobile sur le travail et rapporté par N. Hatzfeld: "c'est moins pénible, mais c'est plus chargé.» (Hatzfeld, 2004). Les améliorations peuvent tout à fait contribuer à diminuer des pénibilités correspondant à des gestes qui sollicitent beaucoup sans avoir un rendement aussi prononcé. Mais la succession des améliorations augmente la mobilisation de l'activité en faisant disparaître des moments qui, du point de vue de l'activité humaine, étaient des occasions de relâcher l'intensité et de se reprendre. Mais le coût n'est pas que physique. Comptent tout autant des formes d'empêchement très sollicitantes pour l'engagement réflexif dans l'activité (impossibilité d'ajuster le mode opératoire, de venir en aide à des collègues et de déployer son idée de la coopération et de la solidarité dans une équipe, etc.).

Le lean fonctionne de ce point de vue sur une représentation implicite du travail qui le rabat purement et simplement sur le fonctionnement des installations. L'activité de travail ne fait, en réalité, l'objet d'aucune conceptualisation spécifique, qui obligerait à penser les exigences dont cette activité pourrait être porteuse et qui ne s'aligneraient pas toujours sur les exigences du rendement des installations.

On retrouvera tous ces constats dans la cartographie du flux, un autre des outils du lean, comprise comme un travail sur l'implantation d'atelier pour éliminer les transports considérés comme improductifs, les stockages, les manutentions et, plus généralement, tout geste inutile (schéma 3 et 4). Dans un process manufacturier, la production correspond très 
largement au flux par lequel les composants circulent de poste en poste pour y faire l'objet d'opérations de transformation et d'assemblage. De tels moments seront effectivement répertoriés comme productifs tandis que les simples circulations et plus encore les temps d'attente feront figure d'opérations sans réel ajout de valeur. Le lean incite à ne pas voir un état de fait inévitable dans l'existence de temps improductifs et, par ailleurs, à rechercher les liens avec l'organisation spatiale des ateliers. À nouveau, il s'agira de dire que, en prêtant attention aux réalités les plus ordinaires du fonctionnement de ces ateliers, on est en mesure d'identifier des améliorations à apporter qui permettront d'économiser des déplacements.

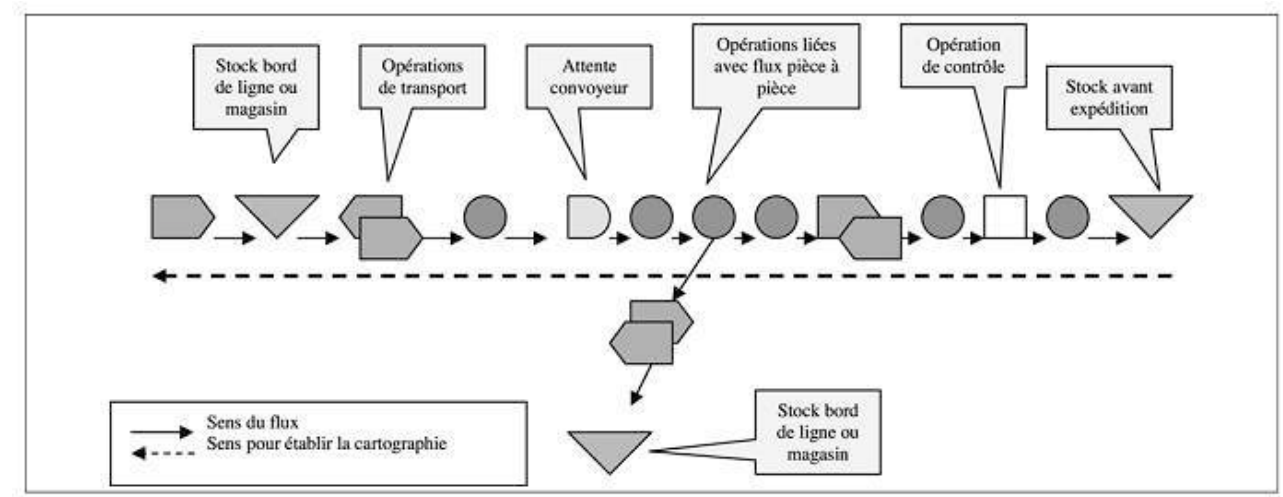

Figure 3 : La cartographie du flux

Figure 3: Flow mapping

Source : Lasnier (2007), p. 102.

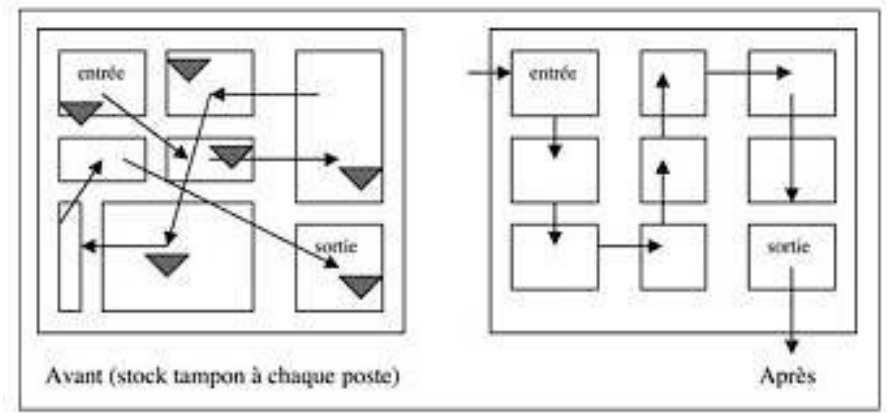

Figure 4 : Réimplantation d'un atelier pour éliminer les temps inutiles

Figure 4: Relocation of a workshop to eliminate timewasting

Source : Lasnier (2007), p. 102.

On n'est guère éloigné des principes ayant inspiré l'importation du dispositif du convoyeur dans les usines de Ford. On est également là face à l'une des répercussions fortes du lean sur l'activité, avec, de nouveau, une optimisation qui interdit le relâchement et la mobilisation de ce que les ergonomes ont coutume d'appeler les «marges de manœuvre ». Pour l'ergonome ou le psychologue du travail, ce temps où l'on n'est pas directement en train de faire n'est pas pour autant un temps où l'on ne fait rien, ne serait-ce que récupérer (mais aussi coopérer, se projeter dans le reste de l'activité, etc.). Dans une démarche lean, ce type de considération n'a guère sa place.

\section{3.- Incompréhension de l'activité ou attention pragmatique au travail ?}

Si l'on résume : au sens propre, il n'y a pas de théorie explicite de l'activité chez Ohno, ni même de théorie du travail. Le SPT est centralement une théorie de l'optimisation de la production où les considérations relatives au travail sont induites plus qu'elles n'ont été 
structurantes dans la construction du schéma intellectuel et des dispositifs. Ces principes et méthodes d'optimisation portent avec eux une mise sous tension de l'activité, pour laquelle, au minimum, on ne pense pas le besoin de relâchement ni de projection dans la tâche à venir et d'organisation personnelle et collective, bref l'enjeu des «marges de manœuvre ». La réflexion d'Ohno est à ce point préoccupée de l'ingénierie de la production que le travail, défini en creux, n'apparaît que comme devant se plier à celle-ci : « Il peut arriver, cependant, que des opérateurs soient empêchés, par des piliers ou des équipements, de voir [= à l'avance] la voiture pour laquelle ils ont un sous-ensemble à préparer. » (Ohno, 1978, p. 62) A ce moment précis, selon lui, les opérateurs de la ligne de sous-assemblage n'ont néanmoins besoin d'être informés que de ce qu'ils ont à faire sur la voiture sur laquelle ils interviennent, sans se préoccuper à l'avance de celle qui lui succédera et «encore moins des suivantes ». Il affirme de manière très tranchée: "Trop d'informations entraînent des anticipations excessives et peuvent causer des confusions dans les séquences opératoires. »

Le travail n'existe pas, dans sa construction intellectuelle, comme cette activité que les sujets ne pourraient accomplir qu'en l'investissant par des processus aussi actifs que ceux de la tâche redéfinie, dans laquelle l'opérateur construit ce qui lui paraît signifier réaliser la prescription (Leplat, 1997). Le travail ne reçoit pas chez Ohno de définition positive. Il est, en fait, l'objet d'une conceptualisation faible. Il y a un concept du gaspillage, à l'égard de quoi le travail est une catégorie induite ou résiduelle, mais à tel point qu'il serait difficile d'en parler comme d'un concept :

«Si l'on dénomme travail, le travail qui est rigoureusement nécessaire pour produire et le reste gaspillage, on peut considérer que l'équation suivante s'applique à chaque opérateur comme à l'ensemble d'une ligne : Capacité = Travail + Gaspillage. » $($ Ohno, 1978, p. 32)

Le gaspillage se définit comme des productions excessives (soit, par conséquent, du travail engagé inutilement), des attentes, des transports et manutentions inutiles, des usinages inutiles, des stocks, mouvements inutiles, productions défectueuses, les fameux mudas.

D'un autre côté, la doctrine d'Ohno, c'est aussi toute une place donnée aux problèmes ordinaires du travail, mais, d'une certaine manière, dans le but que leur reconnaissance officielle évite que les opérateurs n'aillent d'eux-mêmes improviser des solutions :

«Si une machine ou une pièce sont réparées sans que la direction ne soit informée de l'incident, on risque l'improvisation, et les conditions d'une amélioration durable ont peu de chance d'être réunies. Le fait que la machine s'arrête ostensiblement lorsque l'incident se produit permet, au contraire, d'attirer l'attention de toutes les personnes concernées.» (Ohno, 1978, p. 19).

L'andon, cette possibilité donnée à l'opérateur d'interrompre sans autorisation de la hiérarchie le fonctionnement en tirant sur un cordon ou l'arrêt automatique des machines, est une reconnaissance des réalités vécues au plus près de la ligne que des ingénieurs, penchés sur des schémas à plus grosse maille, pourraient négliger comme n'étant que des détails, des frictions ou frottements ne mettant pas en cause la validité des calculs les plus généraux. Le dispositif force la maîtrise et les cadres à se pencher sur des problèmes qui empêchent les opérateurs de tenir la cadence et sur lesquels, en dehors de cela, ils ne daigneraient pas se pencher. Street-level bureaucrats dit Lipsky pour évoquer ces fonctionnaires avec lesquels nous interagissons dans les circonstances les plus quotidiennes et pour signaler que l'action publique finit de se faire à leur niveau (Lipsky, 1980); on pourrait dire que le toyotisme invite les ingénieurs à aussi resserrer la maille de leurs raisonnements à celui des line-level workers, ces travailleurs que l'on trouve au pied de la ligne de montage.

Ce sont des principes d'attention au pragmatique ou à l'ordinaire. Comme l'écrit Lasnier, « [d]ans un système lean manufacturing, la contribution humaine sera valorisée, les tâches effectuées par les opérateurs feront l'objet d'analyses, les activités hommes et moyens de production seront coordonnés de manière à apporter un maximum de valeur ajoutée au 
système, des postes ergonomiques permettront de produire des quantités adaptées à la demande du client, au rythme défini par le takt time, le management visuel sera privilégié de manière que l'opérateur dispose des informations utiles pour réaliser des produits conformes. » (Lasnier, 2007, p. 106). Priorité est donnée à l'attention à l'ordinaire et aux solutions faciles à manipuler (management visuel...). En cela, du reste, le lean est très opposé à Six Sigma, solution d'amélioration continue très instrumentée, sophistiquée, avec lequel il est cependant souvent confondu ${ }^{3}$. L'andon investit l'agent de base de responsabilités dont les organisations tayloriennes et fordiennes le privaient au bénéfice de la décision hiérarchique : juger de la qualité et des répercussions pour le reste de la production, dont la nécessité et la pertinence d'interrompre le flux, avec pour effet à court terme de casser la cadence et d'abaisser la productivité. Mais cela ne doit pas s'interpréter comme une prise en compte du travail dans sa «fonction psychologique» (Clot, 1999). Et la cadence reste l'obsession. L'andon, par exemple, n'est pas un dispositif qui s'exercerait dans une liberté des opérateurs à l'égard des contraintes de débit, mais, au contraire, sous l'égide des temps standard et du panneau indicateur renseignant d'un seul coup d'œil sur l'état de la ligne et les ennuis qui s'y opposent éventuellement, donc sur la charge et le respect des standards opératoires.

La réalité de cette capacité des problèmes ordinaires du travail à remonter vers l'action des cadres varie, en fait, énormément selon les configurations nationales. Dans un même groupe automobile, par exemple, l'andon conduira effectivement, dans un pays, à un arrêt total de la ligne, en attente de la venue de la hiérarchie pour constater le problème et le prendre en charge, alors que, dans d'autres contrées, certains opérateurs continueront de s'affairer, voire finiront de réaliser leur production.

T. Pardi montre que, au sein d'un même groupe comme Toyota, ses transplants en GrandeBretagne et en France ne déploient pas exactement dans les mêmes conditions le principe de la modification de la tâche standard par le kaizen (Pardi, 2007). Pardi, contrairement à la plupart des études anciennes, prend le temps de décrire la façon dont le kaizen opère. Il met à jour la construction, au fil du temps, de modes de fonctionnement très contrastés : les uns de type bottom-up, les autres de type top down. Dans ce dernier cas, constaté en France, un animateur de groupe, sous l'influence d'objectifs établis par sa hiérarchie, doit quoi qu'il arrive faire émerger une nouvelle conception de la tâche standard, par exemple ajustée à un takt time plus bas. Il va, dans ces conditions, solliciter la coopération des animateurs d'équipe en dessous de lui, qui vont y travailler la plupart du temps sans leurs opérateurs. Les groupes kaïzen sont ad hoc, non inscrits dans le fonctionnement régulier. Dans le schéma inverse, constaté au Royaume-Uni, un opérateur voire l'ensemble de l'équipe communique à son animateur des propositions. L'animateur en évalue l'intérêt et en parle à sa hiérarchie. Si celle-ci accepte la proposition et si la modification n'implique pas une reconception importante de la tâche standard, la feuille de prescription est modifiée, avec copie à la hiérarchie et au spécialiste de la sécurité et des conditions de travail. Celui-ci et la hiérarchie auront, en revanche, à intervenir plus activement si la modification est d'une plus grande importance.

Quoi qu'il en soit, dans la doctrine d'Ohno, il est peu contestable que cela est fait au bénéfice des standards opératoires, dont le rôle est crucial. Le toyotisme peut, en effet, à certains égards, s'interpréter comme une décentralisation de la fonction méthodes, raccourcissant la perception et le traitement de l'écart entre travail réel et travail prescrit. Il y a un principe de révision continuelle des standards à partir de l'idée de ne pas prendre pour

Six Sigma est une méthode s'employant à combattre l'instabilité de la qualité issue des processus de production. Pour garantir la qualité en bout de ligne, il s'agit de tenir les paramètres sous contrôle statistique, aucune composante d'un processus ne devant quitter un intervalle de six fois l'écart type par rapport à la moyenne générale calculée pour le processus. 
inévitable tout ce qui s'impose par un discours faisant référence aux contraintes techniques ou à l'idée qu'il a toujours été procédé ainsi, si, par ailleurs, il s'agit de procédés coûteux.

Womack, Jones et Roos ont tenté de relativiser la critique du «management par le stress » qui perçait déjà lorsqu'ils écrivaient en s'employant à montrer qu'il s'agissait d'une méthode gagnant/gagnant. Ohno en faisait autant, tout comme s'était attaché à le faire l'illustre prédécesseur Taylor. L'argumentation des trois auteurs du MIT, cependant, cesse sur ce registre d'être aussi alimentée par les faits tirés de leur étude comparée des constructeurs qu'elle l'était sur le reste, pour entreprendre, cette fois, de convaincre le management par une rhétorique l'incitant à la modération. Au fond, s'efforcent-ils de démontrer, le management doit savoir que, avec le lean, si les ouvriers ne «se livrent pas entièrement, c'est un désastre ; si les ouvriers ne sentent pas de la réciprocité, ils ne coopèrent pas... » (Womack et al., 1990, p. 121).

En résumé, on est face à une lean production très fermée pour l'activité de travail et qui, cependant, lui offre des ouvertures. Tout dépendra des processus sociaux de construction du lean in situ : place de tel ou tel cabinets-conseils, historique du management, etc. Pour un intervenant comme l'ergonome, cela signifie notamment qu'il interviendra dans un système d'acteurs et d'objets et dans une trajectoire d'entreprise qui modèlent considérablement la façon dont le lean va être compris et développé.

\section{4.- Le lean management et l'activité de travail}

Si le lean intéresse de nouveau les dirigeants d'entreprises et, par contrecoup, les chercheurs, c'est dans la mesure où il s'est diffusé de nouveau sur des bases, cette fois, plus larges que celles de la lean production : passant au lean management, voire, plus simplement, au lean, la méthode prend une portée nouvelle; l'évolution de vocabulaire laisse entrevoir une méthode dont les promoteurs défendent la portée très universelle. Le lean apparaît sur la liste des nombreuses modes managériales, c'est-à-dire des schémas cognitifs et normatifs à partir desquels les dirigeants et l'appareil gestionnaire des firmes appréhendent le monde, l'interprètent, construisent les enjeux auxquels ils estiment devoir faire face et les bonnes manières d'y faire face (Abrahamson et Fairchild, 1999).

Ce faisant, le lean se montre candidat à une application dans des secteurs d'activité et des contextes d'entreprises de plus en plus éloignés d'une configuration productive comme celle de l'industrie automobile. On peut se poser la question de savoir si cela va impliquer l'importation dans ces contextes de modèles de représentation de la production et du travail dans le mouvement même de la diffusion des méthodes lean.

Le degré de généralité du modèle Toyota a toujours été sujet à débat. La question se pose, en premier lieu, sur les configurations productives concernées : on peut se demander si les principes de gestion de production qu'il recouvre peuvent s'étendre au-delà des ateliers de montage ou de production industrielle. Ohno et surtout Womack, Jones et Roos défendent que les principes et la méthode valent pour l'industrie automobile en général et tout domaine de ces entreprises. Mais ils sont plus fragiles sur les exemples qu'ils prennent en dehors d'activités telles que les presses ou le montage.

La question concerne également le passage du champ de la gestion de production à une compréhension plus large du management. Chez Ohno, il est essentiellement question de principes de gestion de production. Dès que l'on passe à l'ouvrage de Womack et ses collègues, il y a tendance à extrapoler vers des principes de management. Cette extension se fait notamment vers l'analyse de la chaîne de valeur.

Avec le lean management, on pénètre sur un terrain moins stable que celui de la lean production. Du système élaboré par Ohno à la lean production, on avait déjà l'effet d'auteurs, chercheurs à la base, mais approchant pour l'occasion le rôle de gourous du management, dont le modèle, parce qu'il intégrait une forte dose normative, pouvait se prêter 
à des interprétations assez variées de la part des firmes. Cette dimension se renforce ici : il s'agit de plus en plus de promouvoir ce qui devrait être plutôt que de décrire un modèle existant.

Mais, de façon plus précise, deux interprétations peuvent être proposées de ce que recouvre, en définitive, le lean management, qui peuvent être compatibles, mais qui ne signifient pas la même chose du point de vue de la prise en compte des réalités sectorielles de l'activité productive.

D'un premier point de vue, on peut considérer le lean management, comparé à la lean production, comme une remontée vers les principes généraux de management, des préceptes sur la bonne façon de gérer des processus productifs et des hommes. Cela relèverait alors d'une séquence qui aurait fait passer du SPT au lean manufacturing, dans une première étape d'extension, à partir de quoi l'extraction de principes et d'outils dans leur dimension la plus générale aurait permis de dégager un modèle encore plus général, mais plus orienté sur les principes de management que de stricte gestion de production: le lean management. S'il s'agit de cela, cela peut renforcer une identité du lean se faisant d'abord à travers la collection d'outils mis à disposition; ces outils correspondraient à une promesse de performance à travers des solutions génériques, transposables à tout contexte d'activité.

Imaginons des tentatives d'application du lean dans des secteurs où la programmation de l'activité productive, la standardisation des tâches et la mesure exacte des temps opératoires perd de son sens, en particulier dans les services. L'économie du temps propre à de tels secteurs est fondamentalement distincte de celle de l'industrie de série et la transposition dans de tels secteurs des principes du lean peut avoir des côtés destructeurs, pour la valeur elle-même : à vouloir éliminer des temps à première vue improductifs, on peut mettre les agents de base en situation de faire des arbitrages défavorables à des actions qui, bien que mal repérées par les instruments de gestion, contribuaient réellement à la valeur du service. Dans ce cas, le lean accentuera les contraintes ressenties par les agents dans la réalisation de leur activité, jusqu'à un sentiment d'empêchement à effectuer le travail à leurs yeux réellement attendu d'eux.

Mais, autre effet tout à fait envisageable de ce même cas d'application du lean dans de tels secteurs, parce qu'un outil comme le calcul du takt time n'y prend pas rapidement sens, certaines entreprises se trouveront à l'oublier au sein de la liste très fournie des outils du lean - qu'il est rare de voir tous appliqués -, même s'il est parfois considéré comme pivot. Cet oubli peut aussi s'inscrire dans une appropriation plus générale du lean consistant à y voir, non pas une méthode structurée autour de la mesure permanente des temps alloués, mais davantage comme une incitation adressée à tous les niveaux hiérarchiques de se réemparer de sujets existant au niveau des personnels de terrain. On peut ainsi avoir affaire à des entreprises de services qui, l'habitude de mesurer les temps n'étant guère installée, se saisissent du lean en y voyant surtout une méthode aidant à faire voir la réalité sous l'angle des gaspillages qui pourraient être réduits, sans mesure précise des temps.

Une deuxième interprétation est que le lean management n'est pas simplement venu de l'extension du lean manufacturing mais que le développement de ce dernier a favorisé une montée d'intérêt de nombreux secteurs d'activité et milieux professionnels pour les principes du lean. Le lean doit sa nouvelle actualité et l'ampleur qu'il a prise dans les années 2000, non pas seulement, au couple des cabinets de conseil et des directions d'entreprises, mais aussi à des réflexions internes à des communautés professionnelles qui, de façon tout à fait autonome, sont, pour leurs propres doctrines professionnelles, allées chercher du côté du lean des principes et des outils qu'elles voyaient comme directement utiles à leur efficacité dans le travail. C'est le cas en particulier du monde des informaticiens et du lean software development ${ }^{4}$. Toute une variété de leans sectoriels a ainsi fleuri qui a fortement contribué à

4 Je remercie Denis Duplan d'avoir attiré mon attention sur ce point. 
l'essor général du lean.

La séquence est, cette fois, un passage du SPT au lean manufacturing, mais rapidement accompagné par des développements parallèles de leans sectoriels (lean logistics, lean office, lean software development...). Le lean management serait alors l'enveloppe globale de tout cela.

Toutes ces formules ne sont pas nées de façon endogène à des groupes professionnels, mais cela veut tout de même dire qu'il a pu y avoir, non de simples projections du lean et de ses principes généraux, mais, à des degrés variables, un travail d'adaptation aux spécificités sectorielles de l'activité. En quelque sorte, une réingénierie du lean. Là encore, les implications ne sont pas déterminées d'entrée de jeu.

Le lean office est, en effet, là pour rappeler que ce travail peut être très minimal : avec lui, le lean est désormais pensé pour les services, sans jamais considérer que les activités de service seraient des espaces de contraintes très distinctes de celles de l'industrie de série au point de rendre non pertinent le transfert des catégories de pensée conçues pour l'industrie. Le lean y est promu, au contraire, sur l'idée que du travail de bureau s'analyse essentiellement comme une entrée d'inputs, une transformation de ceux-ci et une sortie sous forme d'outputs. Dans ce cas, le lean office est réellement porteur de risques pour l'activité de travail, liés au fait qu'il occulte qu'un tel travail est soumis à des objectifs qui relèvent de la production d'outcomes (d'effets plus généraux et plus lointains) dépassant la simple réalisation des outputs ${ }^{5}$.

Dans ses différentes variantes sectorielles, le lean se réduira de plus en plus aux mudas et à un effort pour forcer les personnels et leur encadrement à les détecter et à les combattre, le tout dans une représentation de l'activité productive et de travail (Tableau 4).

\begin{tabular}{|l|l|l|}
\hline \multicolumn{1}{|c|}{ Lean manufacturing } & \multicolumn{1}{|c|}{ Lean office } & \multicolumn{1}{c|}{$\begin{array}{c}\text { Lean software } \\
\text { development }\end{array}$} \\
\hline Surproduction & $\begin{array}{l}\text { Tâches réalisées sans } \\
\text { besoin, par ex. du reporting }\end{array}$ & Trop de fonctionnalités \\
\hline Attentes & $\begin{array}{l}\text { Délais de réponse, attentes } \\
\text { de validation }\end{array}$ & $\begin{array}{l}\text { Retards de livraison des } \\
\text { modules }\end{array}$ \\
\hline Retouches, corrections & $\begin{array}{l}\text { Production de documents à } \\
\text { retoucher }\end{array}$ & Défauts, bugs \\
\hline Micro-mouvements inutiles & Déplacements physiques & Réapprentissage \\
\hline $\begin{array}{l}\text { Processus opératoires mal } \\
\text { adaptés }\end{array}$ & Sur-qualité & Travail partiellement fait \\
\hline Stocks & $\begin{array}{l}\text { Stocks de documents } \\
\text { inutilisés }\end{array}$ & \\
\hline Transport, acheminement & $\begin{array}{l}\text { Flux de documents, de } \\
\text { données }\end{array}$ & \multicolumn{2}{|l}{} \\
\hline
\end{tabular}

Tableau 4 : Lean manufacturing, lean office, lean software development et leurs mudas

Table 4: Lean manufacturing, lean office, lean software development and their mudas

Incontestablement, cela dessine des représentations de l'activité extrêmement restrictives. Dans les conditions pratiques d'application du lean, cela pourra être problématique si l'effort est réellement entrepris d'adapter l'activité de travail réelle à cette fiction. L'effet sera moins délétère si le lean est saisi comme un outil pour travailler sur la partie de l'activité qui relève

5 Voir l'article de C. du Tertre dans ce dossier. 
le plus du schéma de la transformation d'inputs en outputs, sans exclure d'autres démarches. Dans certains contextes d'entreprise, la démarche lean, même sur la base de ces représentations a priori assez pauvres de l'activité productive, sont surtout des prétextes à se réintéresser aux fonctionnements concrets et à mettre à plat ces fonctionnements. Il existe des cas d'entreprises dans lesquels cela permet de mettre en évidence que les personnels en sont venus à être soumis à des tâches, comme la production de rapports chiffrés, demandées par les directions fonctionnelles, mais sans réelle utilité à leur niveau et dans le reste de l'organisation. Dans ce cas, le lean sert à faire le ménage et à rendre acceptables ces révisions à des directions fonctionnelles qui, sans cela, auraient eu le pouvoir d'y résister.

Dans la façon dont le lean est introduit, ne se jouent donc pas exactement les mêmes implications pour le travail. Il semble qu'il y ait, de façon polaire, deux modes d'utilisation du lean.

À l'une des extrémités, on trouve une introduction du lean qui contraindra fortement l'activité de travail en introduisant des modifications traquant les actions réputées improductives jusqu'à interdire le relâchement. Dans d'autres cas, on se trouve devant une application moins soucieuse de comptabiliser à la seconde près les gaspillages et plutôt dans des formes qui revisitent le fonctionnement en en allégeant, dans le domaine tertiaire particulièrement, la contrainte bureaucratique. Dans le lean office, par exemple, on peut certes trouver, cohérent avec le mode précédent, l'idée d'introduire du workflow mais aussi celle d'alléger le reporting, de supprimer des validations hiérarchiques purement bureaucratiques, etc.

Dès lors, les conséquences du lean pour l'activité ne sont pas déterminées a priori.

\section{Conclusion}

La théorie du travail sous-jacente au lean se déduit plus ou moins aisément des écrits existants. Mais un point fondamental est que le lean existe moins en soi que dans des trajectoires d'entreprises qui sont aussi des trajectoires d'interprétation de cette méthode. Il y a, en fait, des usages du lean, pluriels et plutôt indéterminés, aux conséquences plus ou moins sévères pour l'activité. Les situations où le lean s'impose autour de réorganisations de l'espace de travail selon le modèle de l'opérateur tournant mettent ainsi l'activité sous forte tension. L'intervention ergonomique risque d'être sollicitée dans une compréhension assez biomécanique de cette intervention et sous des formes qui pourront donc appeler l'ergonome à rendre possible l'intensification du travail et la réduction des marges de manœuvre, dans une conception très restrictive de l'activité. Dans d'autres cas, le recours au lean se fera en parallèle et en appui d'un certain bon sens dans le management des hommes qui tente certains acteurs des directions depuis, notamment, diverses crises relatives au travail (épisodes médiatisés de suicides...).

Il ne s'agit donc pas de dire que, suivant son mode d'introduction, le lean pourrait s'appliquer avec un visage dur ou un visage humain et des manifestations sévères ou tolérables. L'idée serait plutôt que le lean est forcément interprété et que certaines interprétations retiennent d'abord un principe de mesure des temps alloués et d'autres n'abordent pas l'activité avec la même prévalence de la mesure. Dans le premier cas, le lean comprime parfois fortement les temps impartis jusqu'à négliger, en les considérant comme improductifs, ceux qui servent à des régulations connexes et à des marges de manœuvre. Même dans ce cas, il faut aussi percevoir le lean comme incluant des aspects de simplification du travail et d'allégement d'actes qui coûtaient peut-être plus qu'ils ne rapportaient aux travailleurs. Dans le second cas, la mise à distance de la mesure a des effets ambivalents : la faible prégnance des mesures évite d'avoir des raisonnements fondés sur la seule identification des productions les plus tangibles pour rapidement détecter des déperditions d'énergie sans percevoir toute la perte d'actes productifs qu'occasionnerait le 
rationnement des moyens. Mais la mesure peut aussi faire défaut au moment où il faudrait outiller le raisonnement d'une direction qui entreprend de considérer que du travail où les aspects peu tangibles sont nombreux doit être repris en main en supprimant des latitudes inutiles.

\section{BIBLIOGRAPHIE}

Abrahamson, E., \& Fairchild, G. (1999). Management Fashion: Lifecycles, Triggers, and Collective Learning Processes. Administrative Science Quarterly, 44(4), 708-740.

Beauvallet, G., \& Huy, T. (2009). L'adoption des pratiques de gestion lean. Cas des entreprises industrielles françaises. Revue française de gestion, 35(197), 83-106.

Bertrand, T., \& Stimec, A. (2011). Santé au travail. Voyage en pays de lean management. Revue française de gestion, 37(214), 127-144.

Bourgeois, F., \& Gonon, O. (2010). Le lean et l'activité humaine. Quel positionnement de l'ergonomie, convoquée par cette nouvelle doctrine de l'efficacité ? Activités, 7(1), 136-142.

Bouville, G. (2010). Les effets de la lean production sur la santé au travail et l'absentéisme : les résultats d'une étude de cas rétrospective dans une entreprise de maintenance ferroviaire. In Colloque "Organisation, gestion productive et santé au travail. Association française de sociologie, Rouen, 16-17 décembre.

Clot, Y. (1999). La fonction psychologique du travail. Paris: PUF.

Colin, T., Grasser, B., \& Jacquot, L. (2011). Normalisation du travail par le Lean et injonction à innover : quel rôle pour les managers? In 4e congrès de l'association française de sociologie, Grenoble, 5-8 juillet.

Coriat, B. (1991). Penser à l'envers. Travail et organisation dans l'entreprise japonaise. Paris: Christian Bourgois.

Fairris, D., \& Tohyama, H. (2002). Productive Efficiency and the Lean production System in Japan and the United States. Economic and Industrial Democracy, 23(4), 529-554.

Hatzfeld, N. (2004). L'intensification du travail en débat. Ethnographie et histoire aux chaînes de Peugeot-Sochaux. Sociologie du travail. 46(3), 291-307.

Holweg, M. (2006). The genealogy of lean production. Journal of Operations Management, 25, 420437.

Kamata, S. (1973). Toyota, l'usine du désespoir. Trad. Française, Paris: Demopolis, 2008.

Lasnier, G. (2007). Le lean manufacturing (système de production à haute performance) dans les industries travaillant en juste-à-temps avec flux régulés par takt-time (rythme de la consommation du client). La Revue des sciences de gestion, (223), 99-107.

Leplat, J. (1997). Regards sur l'activité en situation de travail. Contribution à la psychologie ergonomique. Paris: PUF.

Lipsky, M. (1980). Street-Level Bureaucracy. New York: Russel Sage Foundation.

Morvan E., François M. \& Bourgeois F. (2008). Les systèmes productifs "au plus juste" : quelle place pour l'activité et la santé ? In 43e congrès de la Société d'Ergonomie de Langue Française. Ajaccio, 17-19 septembre.

Ohno, T. (1978). L'esprit Toyota. Trad. française, Paris: Masson, 1989.

Pardi, T. (2007). Redefining the Toyota Production System: the European side of the story. New Technology, Work and Employment. 2(1), 2-20.

Pettersen, J. (2009) Defining lean production: some conceptual and practical issues. The TQM Journal. 2l(2), 127-142.

Rinehart, J., Huxley, C., \& Robertson, D. (1994). Worker Commitment and Labour Management Relations under Lean production at CAMI. Relations industrielles / Industrial Relations. 49(4), $750-775$.

Schouteten, R., \& Benders, J. (2004). Lean production Assessed by Karasek's Job Demand-Job Control Model. Economic and Industrial Democracy. 25 (3), 347-373.

Shimizu, K. (1999). Le toyotisme. Paris: La Découverte.

Stewart, P., Durand, J.-P., Lewchuck, W., Yates, C., \& Danford, A. (2006). Les ouvriers de Vauxhall 
face à la lean production. Le Mouvement social, 217, 33-52.

Tertre, C. (du) (1995). La dimension sectorielle de la régulation. In R. Boyer \& Y. Saillard (Eds.), Théorie de la régulation, l'état des savoirs (pp. 313-322). Paris: La Découverte.

Ughetto, P. (2009). Une réorganisation au concret. L'implantation du lean manufacturing comme travail managérial. In XIIes Journées internationales de sociologie du travail, Nancy, 25-26 juin.

Ughetto, P. (2011). Pour ne pas se tromper de gestion de la santé au travail. Les niveaux d'un management attentif au "métier". Revue française de gestion, 37(217), 61-75.

Valeyre, A. (2006). Organisations du travail, conditions de travail et santé au travail dans l'Union Européenne. Rapport pour la DARES: Noisy-le-Grand, Centre d'études de l'emploi.

Vatin F. (2003). Le travail. Économie et physique 1780-1830. Paris: PUF.

Veltz, P., \& Zarifian, P. (1993). Vers de nouveaux modèles d'organisation ? Sociologie du travail, 1, 3-25.

Vidal, M. (2007). Manufacturing empowerment? "Employee involvement" in the labour process after Fordism. Socio-Economic Review, 5, 197-232.

Womack, J.P., Jones, D.T., \& Roos, D. (1990). Le système qui va changer le monde. Trad. française, Paris: Dunod, 1992.

\section{RESUME}

Le lean connaît un renouveau dans les entreprises depuis le milieu des années 2000 et fait naître un débat sur ses conséquences pour le travail. L'article cherche à identifier la façon dont le lean conçoit l'activité de travail. Chez Ohno, le concepteur de la méthode et de ses outils, on ne trouve cependant guère de théorie explicite, pas plus que chez Womack, Jones et Roos, théoriciens de la lean production. L'idée centrale est la lutte contre les gaspillages dans le fonctionnement ordinaire des ateliers. Cela peut aussi bien conduire à revaloriser l'intérêt pour les conditions matérielles de travail des ouvriers que se traduire par une obsession de la réduction des temps jugés improductifs en obligeant l'activité humaine à s'y plier et à s'exercer sans relâchement. Cette relative indétermination vaut d'autant plus que la diffusion du lean au-delà du secteur automobile implique qu'il s'applique dans des contextes productifs très variables.

\section{MOTS CLES}

Lean, lean management, lean manufacturing, travail

\section{REFERENCEMENT}

Ughetto, P. (2012). Le lean : pensée et impensé d'une activité sans relâchement. Activités, 9(2), 148-167, http://www .activites.org/v9n2/v9n2.pdf

Article soumis le 19 mars 2012, accepté pour publication le 10 juillet 2012 